\title{
THE CONTINUED INCREASE OF PRECARIOUSNESS IN WORKING LIVES AMONG YOUNG
}

\section{ADULTS IN SPAIN, 1987-2017}

\section{Mariona Lozano and Elisenda Rentería, Centre d’Estudis Demogràfics}

Between the ages of 30 and 39, many people make important decisions for their lives, such as having children, getting married or settling down in long-term relationships. In Spain, the median age at first birth among women is 30.8, couples get married at 35 on average (35 years old among women and 37.7 among men), and sign a mortgage around 38. Therefore, from an aggregate point of view, all of society has something to win, or lose, depending on how their young adults do during these ages. However, evidence points to a consistent increase of precarious work over time and across generations, especially for women. Spain, together with Poland, recently recorded the highest percentage of temporary employment in Europe. Yet, no exact calculations on the duration of this type of work are available for Spain. This study aims to estimate the length of the time that Spaniards are expected to be engaged in temporary and insecure work during their thirties comparing 22 cohorts, given the importance of this age interval for our societies. We found that time spent in precarious employment doubled between 1987 and 2017 for men and women, and generations born in 1978 spent twice the time in insecure and temporary jobs than those born in 1957. More importantly, women are especially affected by these types of employment conditions, and higher education levels do not seem to protect them from lower quality jobs.

\section{THE RISE OF PRECARIOUS WORK-LIFE EXPECTANCY}

Recent waves of the Spanish Labour Force Survey (SLFS) revealed that unemployment is declining in Spain, and the number of contributors to the Social Security System is increasing. Apparently, this is good news 10 years after the 2008 Great Recession that dramatically hit this country. However, these numbers are hiding another reality: employment is growing, but precarious conditions are as well.

Although there is still no consensus on its definition, "precarious employment" refers to new forms of employment emerging in the 70s. It might be considered a multidimensional construct encompassing dimensions such as level of certainty over the continuity of employment, individual control over working hours or conditions, level of protection (against unemployment or discrimination), and insufficient income or economic vulnerability. Kallberg (2009) referred to precarious employment as uncertain, unpredictable and risky occupations from the point of view of the worker. Nonetheless, precarious employment not only affects workers -their families are damaged too- as it creates insecurity regarding job loss, and it has consequences beyond the labour market.
FIGURE 1. Percentage of unemployment and precarious employment in Spain according to cohort and sex in Spain, 1987-2017

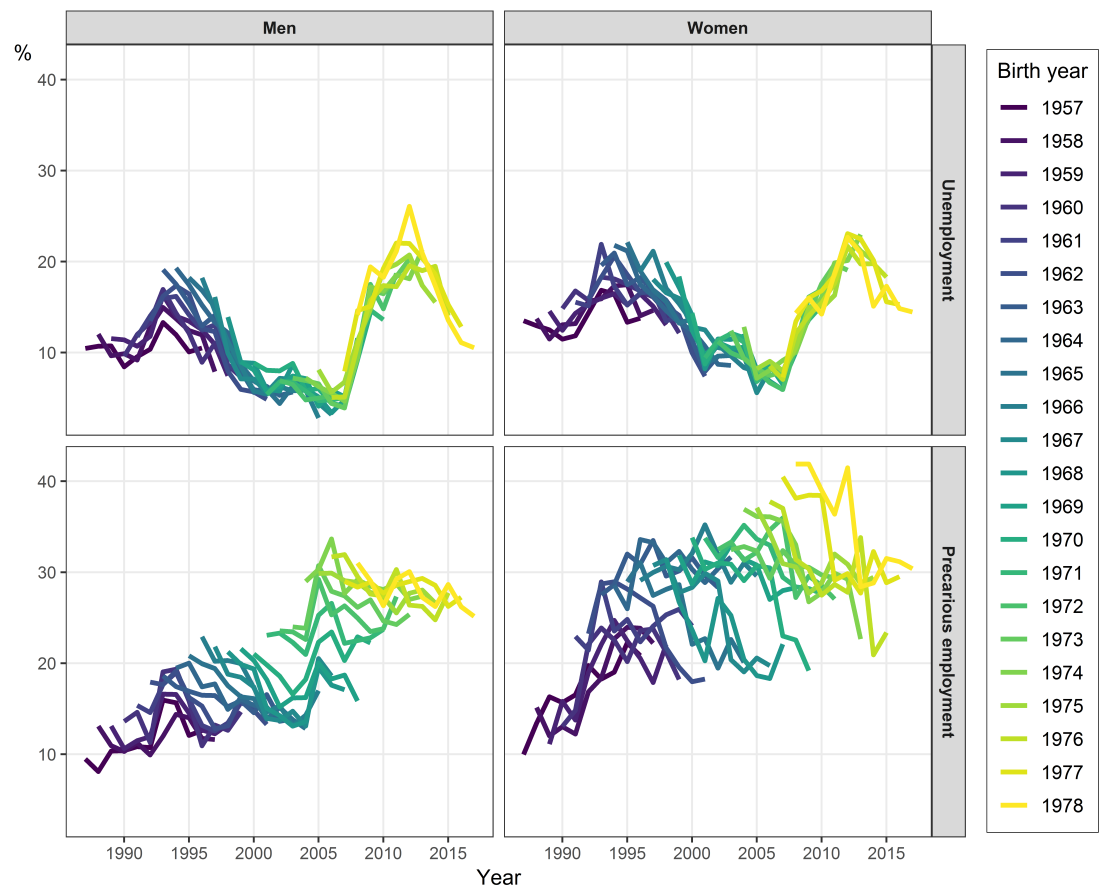

Source: Spanish Labour Force Survey. 
Figure 1 shows unemployment rates and precarious employment (i.e.: long-term temporary job contracts, and unsatisfactory working hours and type of job) for men and women according to their birth year during the last 30 years in Spain. Firstly, unemployment fluctuated over time according to economic cycles. In 1993 and especially in 2008 it grew for both male and female employees. Men seemed to be more affected by the Great Recession, as their rates were higher than women's. This is consistent with past studies that pointed to job loss in typically male industries during the economic crisis, such as construction and manufacturing activities. In addition, unemployment peaked at 26\% in 2013 for men born in 1978 . Later, it decreased, and the youngest male cohorts were placed closer to $10 \%$ and women to $15 \%$. Nonetheless, both levels are still remarkably higher than figures prior to the Recession.

Secondly, precarious employment seemed to be less affected by economic context, and trends indicate a steady rise during the period consideredhere. Nonetheless, duringhighunemployment periods, rates in precarious work decline within generations, meaning that precarious workers were the first expelled from the labour market. Moreover, women's percentages were always higher than men's, and they increased as women's participation rates got higher as well. In other words, during the last three decades, female labour force participation rose considerably, and this took place at the same time that more temporary and fewer secure job contracts were widespread. However, when observing trends within generations, men and women differed

FIGURE 2. Number of expected years in precarious employment between the ages of 30-39 according to sex for observed cohorts

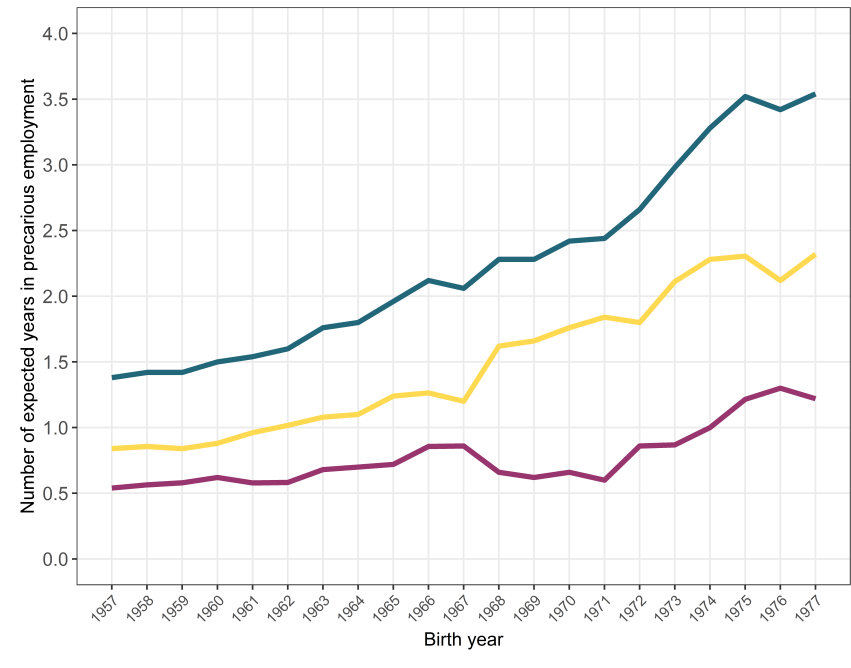

- Men - Women - Gender gap

Source: Spanish Labour Force Survey and Life Tables from the Spanish Statistical Office. considerably. For both sexes, rates of precarious employment declined as they got older; however, the drop among women was more salient, especially among younger generations. In the case of women, this could be explained by a selection mechanism. On the one hand some of these women exited the labour market; on the other hand those who stayed may have transitioned to a more permanent or less insecure position.

\section{PRECARIOUS IS A WOMAN'S NAME}

In Spain, research on the vulnerability of temporary workers and their differentiated patterns of access, progression and exit in the labour market has been extensively developed so far ${ }^{1}$. However, there is a lack of exact statistics on the duration of this type of employment during workers' careers. How long are Spaniards expected to work under precarious conditions? How much have they changed over time? Which generations are more affected by them?

In order to answer these questions, we use the notion of work-life expectancy, which commonly describes the number of years that a person is expected to be economically active; this has the advantage of providing us with a complete picture of the work-life course beyond the specific phases and transitions during the lifespan. To estimate the time in precarious employment, we use data from the SLFS, and the outcome denotes the amount of years that a person between the ages of 30 and 39 at a given year is expected to work precariously. This is shown in figure 2. We observe that both men and women have gained years in precarious work. Men shifted from less than 1 year, between the age interval of 30-39, to slightly more than 2. Figures for women are higher, starting at 1.5 and ending at 2.6 years. In both cases, generations born after 1973 are the ones most affected by this type of employment during their thirties. In addition, for those born in 1978, figures declined in contrast to the immediately preceding generations, but this may be illustrating the effect of the 2008 recession and the rise of unemployment rates.

Gender gap is revealing as well. It compares women's precarious working lives with those of men. The gap between men and women was 1 year for those born in 1957, and almost 2 for cohorts of 1978. That is, between 30 and 39 years old, women born in 1978 experienced two additional years of precarious working conditions tan men born the same year. This increase was parallel to women's entry into the labour market. In 1957 , female activity rate was $45.4 \%$ for those aged 30-39. In contrast, this was $85.1 \%$ in 1970 . Hence, most of the growth in women's employment has been under precarious job conditions. 
In figure 3, we calculate precarious working life according to education. Results indicate that medium levels of education and, in the case of women, also high education, lead to longer time in precarious work. However, this has to be read considering employment rates and socio-economic differences in life expectancy, and entry into the labour market. Highly and medium educated employees have higher participation rates and higher life expectancy, meaning that they are more likely to work and live longer. In addition, the highly-educated also delay their entry into the labour market due to time in schooling, and temporary employment is more likely at early stages of an individual's career. This may explain why medium educated workers recorded the highest number of years in precarious work. They are expected to work longer than low educated on the one hand, and under more temporary conditions than highly educated on the other. However, women's patterns are different, and higher education does not seem to protect them from precarious work.

Highly educated men have gained slightly more than 1 year in precarious employment between the ages of 30 and 39 , and women gained almost 2. For medium education, the increase is 2 years among men and 2.2 for women, and at low education levels it was 2.5 for men and 2 for women. Among men, high levels of schooling seemed to protect them from precarious employment.
However, among women, higher participation rates exposed them to longer years in temporary jobs, and low educated women with lower participation rates in the labour market and lower life expectancy - spent less time precariously employed than other levels of schooling. Finally, differences according to education were more salient among men than women, highly educated men being those who appeared to be the most advantaged, and low educated women the least.

\section{CONCLUSION: YOUNG ADULTS' PRECARI- OUSNESS AFFECTS ALL OF SOCIETY}

In this study we calculated the duration of working life in precarious employment among young adults (30-39 years) for the period 1987 and 2017 in Spain, comparing 22 cohorts born between 1957 and 1978. Results show the relentless increase in the time spent in precarious work. Men born in 1957 spent 0.8 years between the age of 30 and 39 in precarious work, and those born in 1978 spent 2.1 years. For women, figures are higher, shifting from 1.4 to 3.5 years. In addition, gender gap in precarious working life has also increased, especially for those generations born after 1972. These were in their thirties during the early 2000s, when temporary contracts had risen considerably. In addition, during the Great Recession these

FIGURE 3. Number of expected years in precarious employment between the ages of 30-39 according to sex and education level for observed

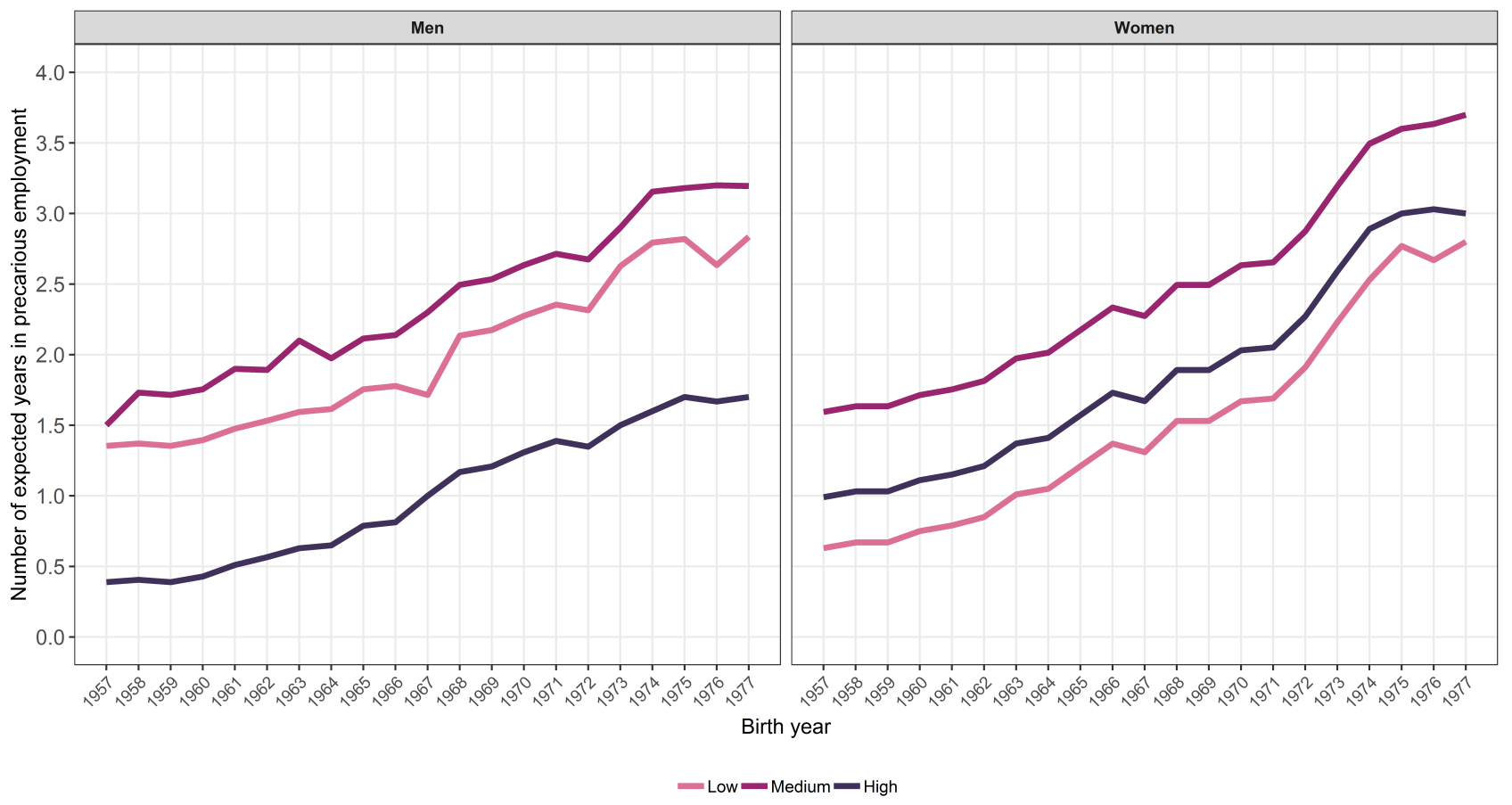

Source: Spanish Labour Force Survey and Life Tables from the Spanish Statistical Office.

Note: Mortality rates for education are not available for the period of time considered here, and therefore our estimates do not consider the probability. 
workers were also the first expelled from the labour market. Even though this is a trend observed all across Europe, temporary rates are among the highest in Spain. What may be the consequences?

First, we focused on young adults, and the results pointed to the accelerating deterioration of their working conditions during their lifespan. Although this is not a new conclusion, we show that time spent in insecurity and temporality has doubled in the last 30 years. Facing long-term uncertainty frustrated young people's entry into adulthood, and it postpones parental emancipation, economic independence, long-term relationships, marriage and fertility. Increased insecurity, especially at these ages, wastes resources that a country like Spain, with a growing ageing population, cannot afford - not in terms of contributors to Social Security systems, or in terms of frustrated parenthood, or individuals' discontent.

Second, vulnerability is especially troubling in the case of women, pointing directly to gender inequality as one of the greatest challenges of our society. Women's labour market participation has been associated with delays in fertility. However, increased job temporality and insecurity hampers it as well. Thus, delays in family formation are also reflecting the rise of precariousness that women especially are experiencing. More importantly, for them, not even the achievement of higher levels of education seems to be protective.
Third, the study shows how economic recessions have diminished working lives. However, while unemployment has fluctuated according to specific circumstances, precarious work seems to be more structural, and it has increased continuously from generation to generation regardless of the economic context. Today, exactly ten years after the Great Recession of 2008, employment is recovering in Spain, but it does so in more precarious conditions.

To sum up, we have observed that levels of unemployment, after the Recession and the implementation of austerity policies, seem to be decreasing, but the real problem is precariousness, especially among women-hence, the importance of taking political action. The best policy to counterbalance the negative effects of high levels of infertility and a growing ageing population is to reduce precariousness. Especially among young adults. Especially among women. A better future is in our hands.
Bibliography
Kalleberg, A. (2009) "Precariou

Work, Insecure Workers: Employment Relations in Transition". American Sociological Review, 74 (1): 1-22.

Miguélez, F. (coord.); Alós, R.; Carrasquer, P.; Lope Peña, A.; Molina, P.; Pastor, A.; Recio, A.; RodríguezSoler, J.; Verd Pericàs, J.M. (2015) Diagnóstico socio-económico sobre las políticas de empleo en España, 2012-2014. Bellaterra: Centre d'Estudis Sociològics QUIT/Institut d'Estudis del Treball.

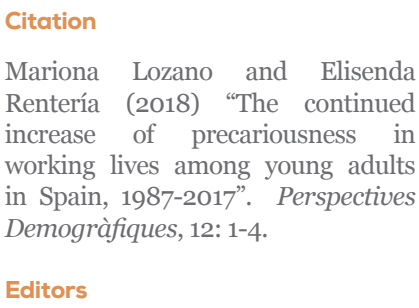

Mariona Lozano and Elisenda Rentería (2018) "The continued increase of precariousness in working lives among young adults in Spain, 1987-2017". Perspectives Demogràfiques, 12: 1-4.

Editors

Andreu Domingo and Albert Esteve Please address correspondence to:

Mariona Lozano mlozano@ced.uab.cat
Contact

Centre d'Estudis Demogràfics. Carrer de Ca n’Altayó, Edifici E2 Universitat Autònoma de Barcelona o8193 Bellaterra / Barcelona Spain

Telephone: +34935813060 Email:demog@ced.uab.cat Web page: www.ced.uab.cat
Graphics: Anna Turu Layout: Xavier Ruiz Vilchez

URL

http://ced.uab.es/difusio/butlleti-perspectives-demografiques 